\title{
A new flap combination for reconstruction of lower nasal dorsum and supra-tip skin defects
}

\author{
Mélanie Guesnier ${ }^{1,2,3}$, Xavier Claveleau ${ }^{1,2}$, Marielle Longeac ${ }^{1,2}$, Isabelle Barthélémy ${ }^{1,2,4}$, \\ Nathalie Pham Dang ${ }^{1,2,4}$, Arnaud Depeyre ${ }^{1,2,5,6}$ \\ ${ }^{1}$ Department of Oral and Maxillofacial, Estaing Hospital, CHU Clermont-Ferrand, Clermont-Ferrand; ${ }^{2}$ University of Auvergne Faculty of \\ Medicine, Clermont-Ferrand; ${ }^{3}$ Department of Dermatology, Estaing Hospital, CHU Clermont-Ferrand, Clermont-Ferrand; ${ }^{4} U M R$ Inserm \\ U1107, Neuro-Dol, Trigeminal Pain and Migraine, Faculty of Dentistry, University of Auvergne, Clermont-Ferrand; ${ }^{5}$ CROC EA 3847 \\ Laboratory, Faculty of Dentistry, University of Auvergne, Clermont-Ferrand; ' INSERM, CHU Lille, U1008-Controlled Drug Delivery \\ Systems and Biomaterials, University of Lille, Lille, France
}

Repairing surgical defects of the nose is still challenging due to its tridimensional shape and its aesthetic concern. Difficulty in reconstructing nasal subunits lies in their contour, skin texture and limited availability of adjacent skin. For lower nasal dorsum and supra-tip regions, we design a new combined local flap as existing local flaps may give disappointing results. This combination flap was performed on two patients for reconstruction of the lower nasal dorsum area after basal cell carcinoma excision. Size of the excision ranged from 20 to 25 $\mathrm{mm}$ diameter and safe margins were obtained. The defects were reconstructed with a local flap that combined a rotation nasal flank flap and a V-Y advancement nasolabial flap. Excision and reconstruction were performed in a one-stage surgery under intravenous sedation and local anesthesia. There were no postoperative complications and no flap loss occurred. Aesthetic and functional results after 6 months postoperatively were satisfying without modification of nasal shape. This flap is reliable and offers interesting functional and aesthetic outcomes. It can be considered as a new reconstruction alternative for supra-tip and lower nasal dorsum skin defects performed in a one-stage procedure under local anesthesia.

Keywords Nose defect / Supra-tip / Aesthetic subunit / Flap

\author{
Correspondence: Arnaud Depeyre \\ Department of Oral and Maxillofacial, \\ Estaing Hospital, CHU Clermont- \\ Ferrand, 1 Place Lucie Aubrac, \\ F-63003 Clermont-Ferrand, France \\ Tel: +33-642-932-001 \\ Fax: +33-473-750-446 \\ E-mail: depeyrearnaud@gmail.com
}

Received: November 15, 2018 • Revised: May 24, 2019 • Accepted: June 13, 2019

pISSN: 2234-6163 • elSSN: 2234-6171 • https://doi.org/10.5999/aps.2018.01354 • Arch Plast Surg 2019;46:480-483

This article was presented at the National French Congress of SFSCMFCO on October 4, 2018, in Marseille, France.

\section{INTRODUCTION}

Repairing surgical defects of the nose after skin cancer excision remains challenging due to the three-dimensional shape of the nose and aesthetic concerns. In the lower part of the nasal dorsum and the supra-tip region, skin defects can be repaired through healing by secondary intention, skin grafts, or preferentially by local flaps, but these techniques sometimes yield disap- pointing functional or aesthetic results [1]. Preserving global facial harmony and respecting the principle of aesthetic subunits developed by Burget and Menick [2] are essential. However, difficulties are posed by the limited area from which to harvest similar skin in terms of thickness, color, and adnexal composition [2-4]. We have specifically focused our attention on the supra-tip region. Many local flaps for this region can be used, such as the Rieger-Marchac and Rintala flaps, but we have experi- 
enced some technical difficulties and questionable results with those flaps [5]. Therefore, we present an interesting alternative: a surgical technique that combines a rotation nasal flank flap and an advancement nasolabial flap for the reconstruction of supra-tip and low nasal dorsum defects.

\section{IDEA}

The case 1 in which we used this technique was a 45 -year-old woman who presented with an invasive basal cell carcinoma at the low-mid nasal dorsum. A surgical excision with a 7-mm margin was recommended based on the pathology results (Fig. 1A). After resection, the skin defect measured $20 \times 22 \mathrm{~mm}$ and was successfully reconstructed using our local flap.
The case 2 was an 82-year-old who man presented with a nodular basal cell carcinoma at the low nasal dorsum that was slightly lateralized to the right side. A surgical excision with a 5-mm margin was recommended based on the pathology results (Fig. 2A). He had thrombocytopenia associated with lymphoma and needed platelet transfusion before surgery. The post-resection skin defect was $25 \times 20 \mathrm{~mm}$ and was reconstructed using our local flap.

All surgical procedures were performed under intravenous sedation and local anesthesia. Each lesion was first carefully delimited before removal and then excised with the recommended margins. Once removed, the resected specimens were sent to the pathology department to identify marginal involvement. When they were declared free, reconstruction was started. After

Fig. 1. Intraoperative pictures of case 1

(A) Preoperative flap design and resection margins. (B) Prepared flap. (C) Immediate operative state.
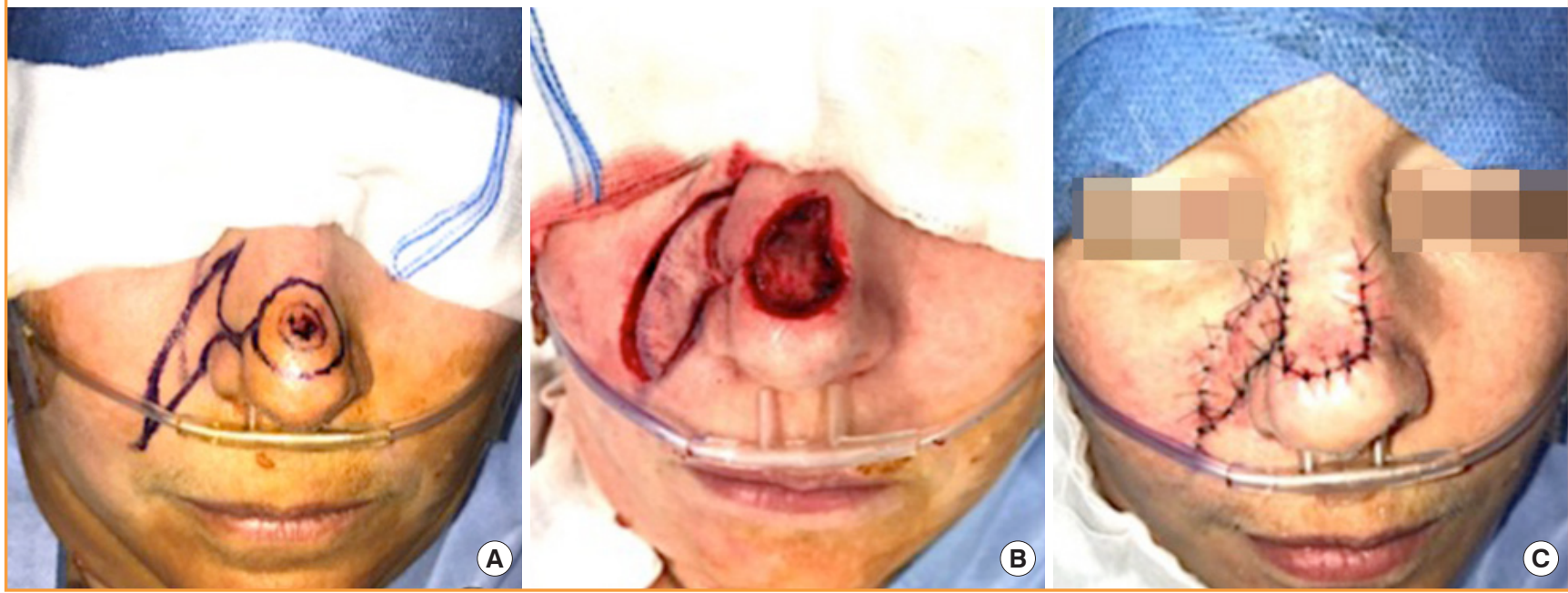

Fig. 2. Intraoperative pictures of case 2

(A) Preoperative flap design and resection margins. (B) Prepared flap. (C) Immediate operative state.
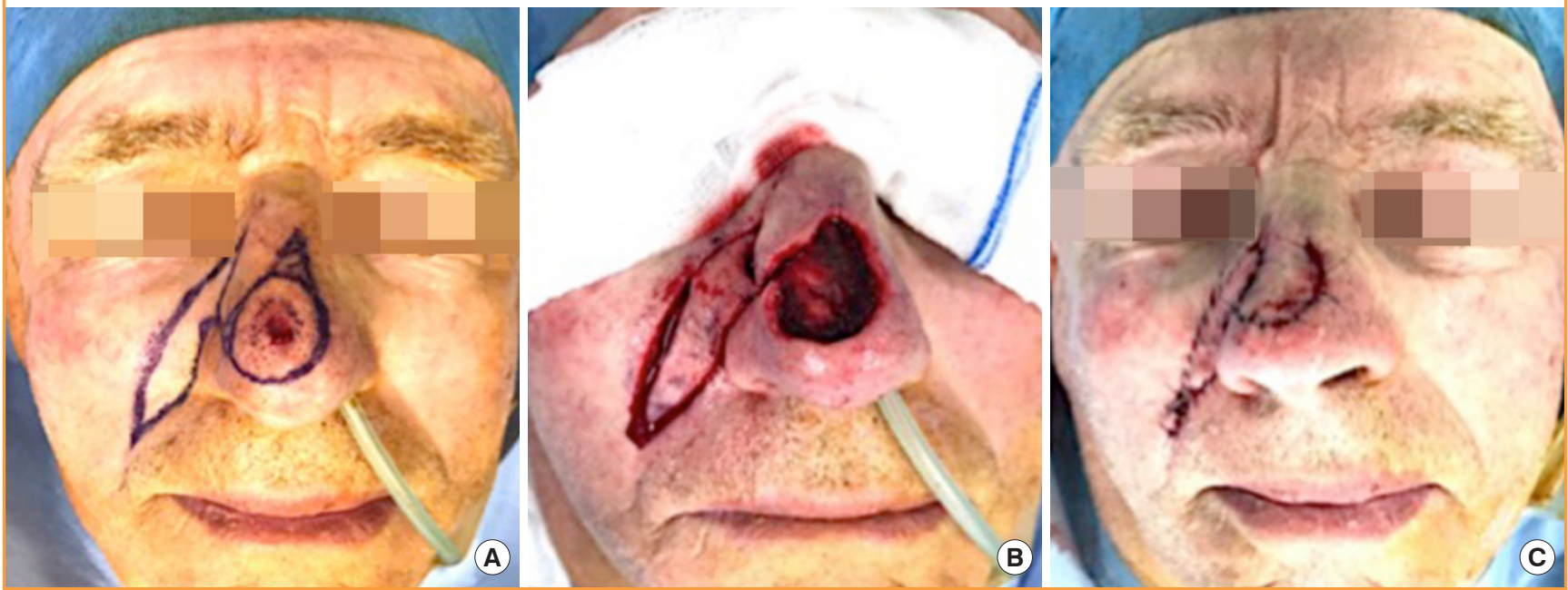
infiltration with lidocaine and 1:200,000 epinephrine, an incision was made with a No. 15 blade through the skin and the superficial muscular layer. Rotation nasal flank flap dissection was strictly performed in the plane above the periosteum and perichondrium of the lateral nasal flank. Incision and dissection did not involve the alar rim region, which was left fully intact. A contralateral back cut at the top of the nasal flank flap was performed to ensure that the Sheen lines and aesthetic subunits were well preserved, as well as to increase the mobility of the flap. Next, a classical advancement nasolabial flap was designed to close the harvesting area of the rotation nasal flank flap, which was strictly above the alar rim (Figs. 1B, 2B). The rotation nasal flank flap was turned medially and the nasolabial flap was advanced. The sutures were performed in two layers: one layer of interrupted inverted stitches with a 4-0 absorbable suture and one layer on the skin with a 5-0 non-absorbable suture (Figs.

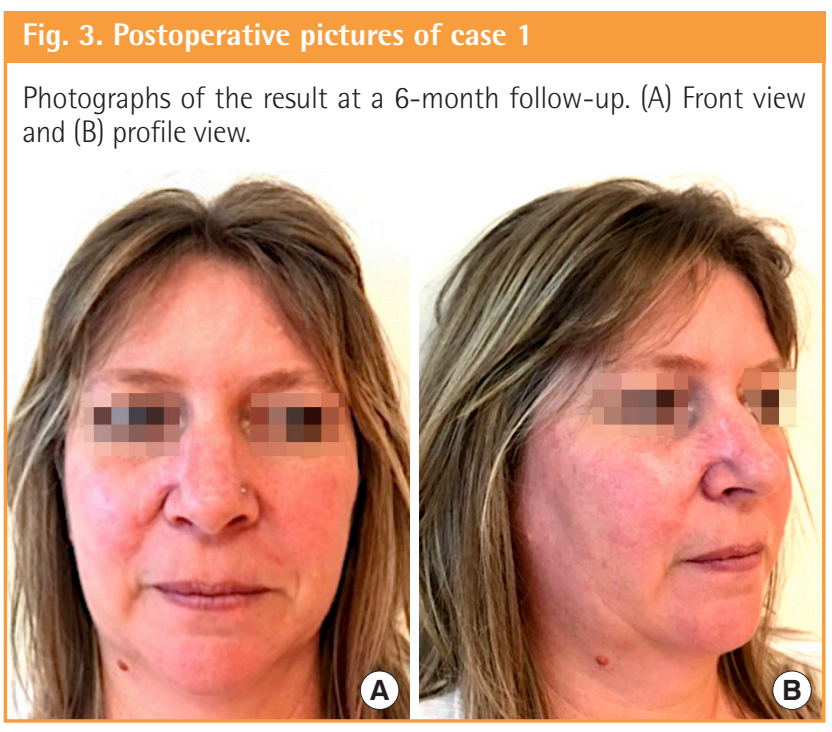

Fig. 4. Postoperative pictures of case 2

Photographs of the result at a 6-month follow-up. (A) Front view and (B) profile view.
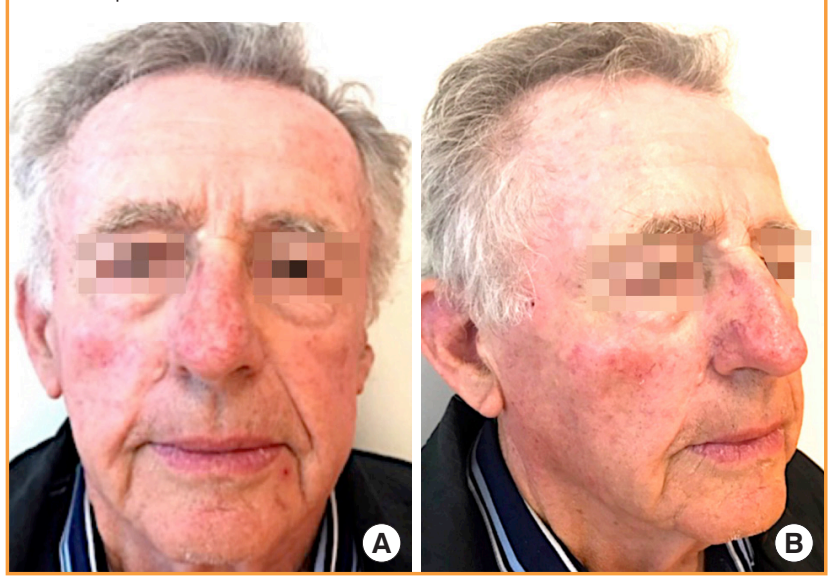

$1 C, 2 \mathrm{C})$. The stitches were removed after 8 days. There were no postoperative complications, and in particular there was no secondary infection or flap necrosis. The patients were followed until 6 months postoperatively. The scars were acceptable and there was no modification of their nasal shape (Figs. 3, 4).

\section{DISCUSSION}

The difficulty in reconstructing nasal subunits lies in their contour, skin texture, and the limited availability of adjacent skin. Using a skin graft often results in an aesthetically poor result, with problems including differences in skin texture and a depression scar [6]. The use of dorsal nasal flaps for nasal dorsum or supra-tip reconstruction is not innovative. Rintala, Rieger, and later Marchac previously demonstrated interesting results with such flaps $[7,8]$. Specific criteria are required for reconstruction using these local flaps to have a high likelihood of success, including a defect diameter $\leq 2.0 \mathrm{~cm}$, no defect extension inferior to the tip-defining points, and location $\geq 0.5 \mathrm{~cm}$ from the alar rim [9].

The lower the location of the skin defect on the nasal dorsum, the less satisfying results are obtained [4]. Using nasal dorsal flaps in such cases can cause partial flap necrosis and a slight cephalic rotation of the nasal tip, such as upturned nose or short nose. This is due to the lack of cutaneous laxity in the nasal dorsum [10]. Moreover, because of the glabellar incision, a visible scar is created, which may lead to an unattractive reduction of the distance between the eyebrows [9].

\section{Fig. 5. Schematic drawings of the combination flap}

The limits of the flaps strictly preserve the nasal subunits and Sheen lines. Nasal subunits from Burget [2]: dorsum (1), lateral walls (2), tip (3), alar lobules (4), soft triangles (5), columella (6). The Sheen lines (SL) are shown in blue, the oncological excision region in red, and the limits of the flaps in green ( $A^{\prime}$ : rotation nasal flank flap; $B^{\prime}$ : advancement nasolabial flap). (A) Markings of the flaps before excision. (B) Flaps sutured together after excision and reconstruction.

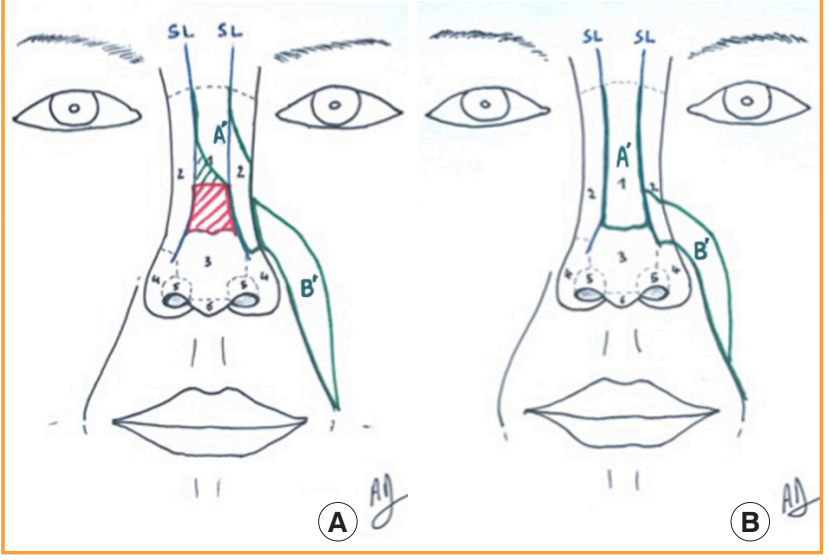


The local combination flap reported in this procedure (Fig. 5) offers significant advantages in terms of functional and aesthetic results. There is no cephalic rotation of the tip and no impact on the direction of nares opening. In fact, the nasal flank rotation flap design descends to the nasal ala and recruits a large lateral nasal skin area, permitting tension-free closure. An advanced nasolabial flap reconstructs the nasal flank skin defect, and sutures are performed to preserve the nasal subunits and Sheen lines. In particular, the nasal ala remains perfectly intact with this technique, which ensures a suitable aesthetic outcome [11]. Moreover, it avoids a glabellar scar and eyebrow dystopia.

None of the patients suffered from flap necrosis, as this flap seems to have good vascular reliability from the upper nasal dorsum and inner canthus area, with blood supply from the angular and external nasal branches of the ophthalmic and contralateral facial arteries [4].

In addition to these good results, this local flap is relatively easy to perform in a one-stage surgical procedure under local anesthesia. In our experience, it appears important to ensure a secure pathological surgical resection through a frozen-section histological examination before considering the reconstruction step.

This combination flap is reliable and offers interesting functional and aesthetic outcomes, with no long-term changes of nasal shape. It can be considered as a new reconstruction alternative for supra-tip and lower nasal dorsum skin defects. The next step is to harvest these flaps as perforator flaps based on the lateral nasal and upper dorsal nasal arteries [12].

\section{NOTES}

\section{Conflict of interest}

No potential conflict of interest relevant to this article was reported.

\section{Ethical approval}

The study was approved by the Institutional Review Board of CPP SUD-EST VI Clermont Ferrand Universitary Hospital (IRB No. 2019/CE 25) and performed in accordance with the principles of the Declaration of Helsinki. Written informed consents were obtained.

\section{Patient consent}

The patients provided written informed consent for the publication and the use of their images.

\section{Author contribution}

Data curation, formal analysis: Guesnier M, Claveleau X, Longeac M, Depeyre A. Conceptualization: Guesnier M, Longeac M,
Depeyre A. Funding acquisition, project administration: Guesnier M, Claveleau X, Depeyre A. Methodology: Guesnier M, Depeyre A. Visualization: Guesnier M, Claveleau X, Barthélémy I, Dang NP, Depeyre A. Writing-original draft, review \& editing: Guesnier M, Claveleau X, Barthélémy I, Dang NP, Depeyre A.

\section{ORCID}

Mélanie Guesnier https://orcid.org/0000-0003-3214-8563

Xavier Claveleau https://orcid.org/0000-0002-9797-6039

Marielle Longeac https://orcid.org/0000-0001-6104-040X

Isabelle Barthélémy https://orcid.org/0000-0002-1569-4231

Nathalie Pham Dang https://orcid.org/0000-0002-0385-1981

Arnaud Depeyre https://orcid.org/0000-0002-7027-2947

\section{REFERENCES}

1. Rogers-Vizena CR, Lalonde DH, Menick FJ, et al. Surgical treatment and reconstruction of nonmelanoma facial skin cancers. Plast Reconstr Surg 2015; 135:895e-908e.

2. Burget GC, Menick FJ. The subunit principle in nasal reconstruction. Plast Reconstr Surg 1985;76:239-47.

3. Singh DJ, Bartlett SP. Aesthetic considerations in nasal reconstruction and the role of modified nasal subunits. Plast Reconstr Surg 2003;111:639-48.

4. Chaput B, Lauwers F, Lopez R, et al. Nose surgical anatomy in six aesthetic subunits. Ann Chir Plast Esthet 2013;58: 132-45.

5. Eren E, Beden V. Beyond Rieger's original indication; the dorsal nasal flap revisited. J Craniomaxillofac Surg 2014;42: 412-6.

6. Branham GH. Nasal reconstruction. Facial Plast Surg 2017; 33:1-2.

7. Rieger RA. A local flap for repair of the nasal tip. Plast Reconstr Surg 1967;40:147-9.

8. Marchac D, Toth B. The axial frontonasal flap revisited. Plast Reconstr Surg 1985;76:686-94.

9. Rohrich RJ, Muzaffar AR, Adams WP Jr, et al. The aesthetic unit dorsal nasal flap: rationale for avoiding a glabellar incision. Plast Reconstr Surg 1999;104:1289-94.

10. Gurunluoglu R, Williams SA. Nasal reconstruction after malignant tumor resection: an algorithm for treatment. Plast Reconstr Surg 2011;127:1387-8.

11. Jeong HH, Choi DH, Hong JP, et al. Use of a helical composite free flap for alar defect reconstruction with a supermicrosurgical technique. Arch Plast Surg 2018;45:466-9.

12. Lombardo GA, Tamburino S, Tracia L, et al. Lateral nasal artery perforator flaps: anatomic study and clinical applications. Arch Plast Surg 2016;43:77-83. 